\title{
Giant seminoma in an undescended testicle metastasizing to the neck and liver
}

\author{
KAI-MIN GUO ${ }^{1}$, YANG LIU ${ }^{2}$, YAN-PING ZHONG $^{3}$ and HONG-LIANG WANG ${ }^{1}$ \\ Departments of ${ }^{1}$ Andrology, ${ }^{2}$ Radiology and ${ }^{3}$ Pathology, \\ The First Hospital of Jilin University, Changchun, Jilin 130021, P.R. China
}

Received October 8, 2015; Accepted March 2, 2016

DOI: $10.3892 / \operatorname{mco} .2016 .829$

\begin{abstract}
Germ cell tumors account for $98 \%$ of all testicular malignancies. Delays in seeking treatment are unfortunately common and may lead to metastatic spread. The present study reported a case of a 24-year-old man with a giant $12 \times 10 \mathrm{~cm}$ left inguinal mass and a left neck mass that had grown rapidly during recent months. Computed tomography confirmed that the mass measured $12.1 \times 9.4 \mathrm{~cm}$ and was a left undescended testicle malignancy, and also revealed widespread metastasis to the liver and a large retroperitoneal mass $(12.6 \times 8.2 \mathrm{~cm})$. Immunohistochemical staining confirmed seminoma. The patient was treated with chemotherapy with the VIP protocol (cisplatin, etoposide and ifosfamide). Following courses of chemotherapy, the patient received complete clinical remission and was disease-free at the 6 month follow-up.
\end{abstract}

\section{Introduction}

Germ cell tumors account for $98 \%$ of all testicular malignancies. Testicular cancer represents the most common malignancy in males aged between 15 and 34 (1). Cryptorchidism remains the best established risk factor for testicular germ cell tumors. It is estimated that a cryptorchid testicle is $30-50$ times more likely to develop a malignant neoplasm compared with a normally placed organ. The incidence is higher even following orchiopexy, if done after 6 years of age. Notably, molecular studies have shown strong evidence of an association between genetic alterations and testicular germ cell tumors (2)

It is fortunate that testicular germ cell tumors represent a highly curable malignant tumor entity, even in the presence of metastasis. The overall survival rate is $\sim 90 \%$, considering all stages are reported (3). In the following case report,

Correspondence to: Professor Hong-Liang Wang, Department of Andrology, The First Hospital of Jilin University, 71 Xinmin Street, Changchun, Jilin 130021, P.R. China

E-mail:wh11228@126.com

Key words: cryptorchidism, chemotherapy, complete remission, late metastasis, seminoma a 24-year-old man is presented in severe condition with a giant $12 \times 10 \mathrm{~cm}$ left stage III cryptorchid seminoma metastasizing to the neck and liver. Chemotherapy yielded complete remission of the primary lesion.

\section{Case report}

In Sepember 2014, a 24-year-old previously healthy man accompanied by his parents, was admitted to Department of Otolaryngology, The First Hospital of Jilin University (Jilin, China), complaining for a left neck mass that had grown rapidly during recent months. The patient also complained of a huge mass located in the left groin. However, it had failed to draw his attention due to non-tenderness and its hidden nature. He had no hematuria, no fever, chill or rigors, with the exception of weight loss during the past year.

Upon examination, the patient was emaciated, nearly in a state of cachexia, at $<45 \mathrm{~kg}$. A $3.0 \times 3.0 \mathrm{~cm}$ mass situated in left supraclavicular fossa extending to the lower cervical region was palpable. Abdominal examination revealed a left inguinal mass of $12 \times 10 \mathrm{~cm}$, directly beneath the skin, extending to the left rib, hard in consistency. Scrotal examination revealed the absence of the left testicle and normal scrotum. The patient was then transferred to the Department of Andrology.

Computed tomography (CT) confirmed that the mass measuring $12.1 \times 9.4 \mathrm{~cm}$ was a left undescended testicle malignancy, and revealed widespread metastasis to the liver and a large retroperitoneal mass $(12.6 \times 8.2 \mathrm{~cm}$; Fig. 1). The tumor markers $\alpha$-fetoprotein, the $\beta$-subunit of human chorionic gonadotrophin and lactic dehydrogenase were within normal limits. All laboratory tests, including complete blood count, biochemistry and chest X-ray, were normal. The provisional diagnosis was testicular metastatic tumor, with a suspicion of lymphoma or germ cell tumor.

The needle biopsy of the supraclavicular mass was performed. Pathological examination revealed nodules of metastatic tumor cells lined in the pattern of nests and cords, divided by intervening fibrous septa with a moderate lymphocytic infiltrate. Immunohistochemical stains revealed diffuse expression of placental alkaline phosphatase and OCT3/4. In addition, the tumor cells exhibited strong and diffuse positivity for cluster of differentiation (CD)117 and D2-40, which confirmed the seminoma. The proliferation marker, Ki-67, revealed a high proliferation index ( $>30 \%$; Fig. 2$)$. 

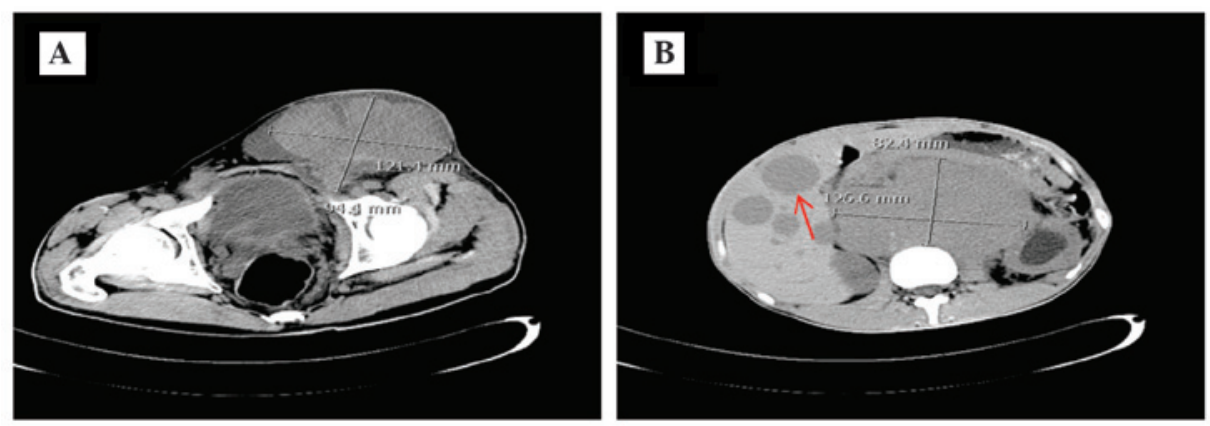

Figure 1. Computed tomography of the abdomen and pelvis revealed (A) a giant left inguinal testicular tumor with (B) widespread metastasis to liver (red arrow) and a large retroperitoneal mass.
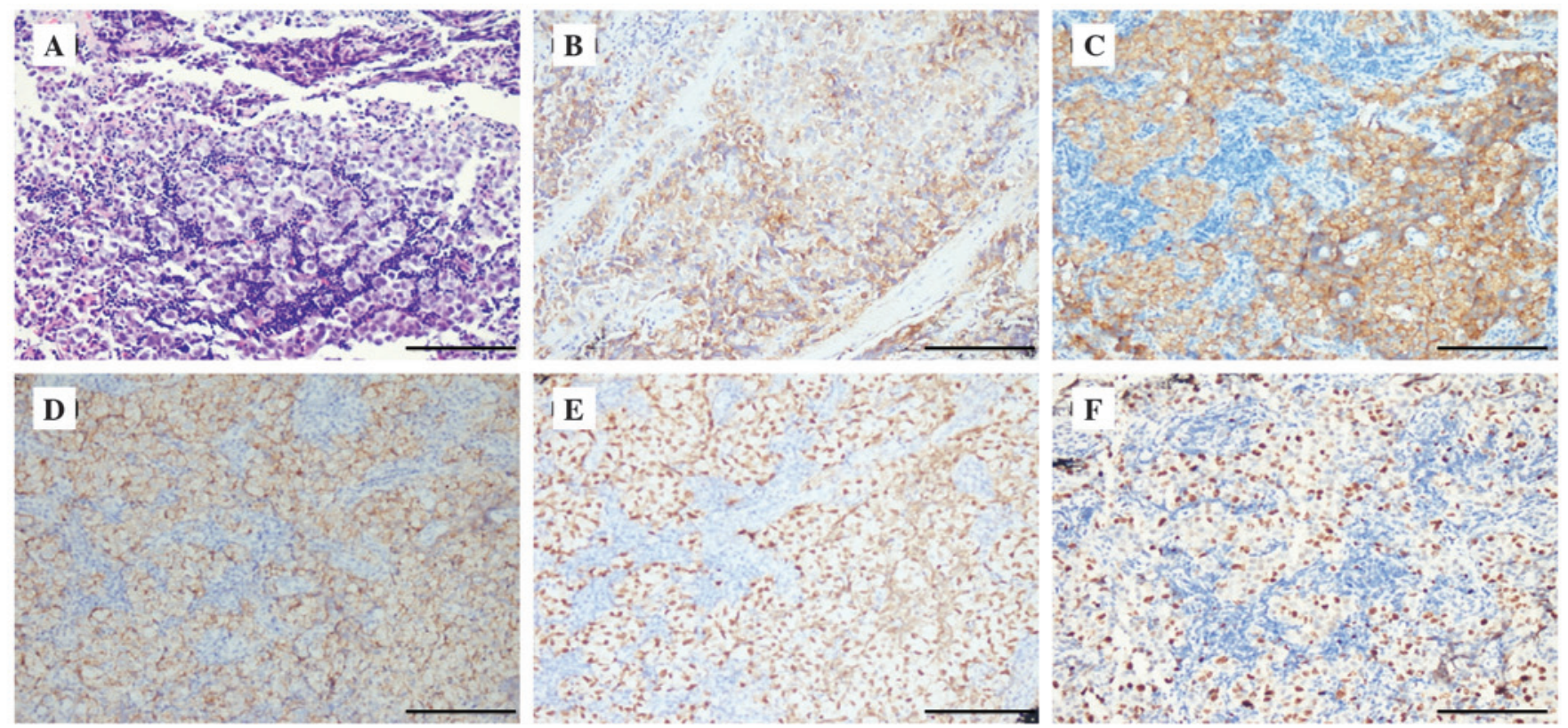

Figure 2. Histology and immunohistochemistry of the cervical node. (A) Tumor cells were stained with hematoxylin and eosin staining and appeared to be arranged in a nest and cord pattern, with lymphocyte infiltration. Immunohistochemistry revealed that the tumor cells exhibited (B) cytoplasmic and cytomembrane positivity with PLAP, (C) positivity with cluster of differentiation 117, (D) positivity with D2-40 and (E) cytonucleus positivity with OCT3/4. (F) Ki-67 revealed a high (30\%) proliferation rate (magnification, $\mathrm{x} 100)$.

Due to the extent of the metastasis, the tumor was considered inoperable, both oncologically and in view of the resulting morbidity. At the interdisciplinary tumor board, chemotherapy with curative intent was indicated. Finally, the patient was administered the chemotherapy VIP regimen (four cycles of cisplatin, etoposide and ifosfamide). Six months after this routine therapy, the patient remained stable and achieved complete remission. CT revealed a reduction of the inguinal mass by $98 \%$ without changes in the size of the retroperitoneal lymph nodes. The patient was informed about surgical resection of the left testicle, however, he refused further surgery due to personal reasons.

\section{Discussion}

Testicular cancer is the most common cancer type in men between the ages of 20- and 35-years old. Seminoma is the most common of the testicular germ cell tumor types, accounting for $\sim 30 \%$ of all testicular neoplasms. The undescended testicles harbor a 20-48 times higher potential for malignant transformation compared with the normally descended testicles. The relative risk of malignancy is highest for the intra-abdominal testis (5\%) and is significantly lower for the inguinal testis (1.25\%) (4). Metastases of seminoma are rare, unless it is associated with sarcoma (5). In the review by van Vledder et al (6), $4 \%$ of patients with seminoma had cervical metastasis. The peak age for presentation is 30-35 years for classic seminoma, secondary peaks are noted in infancy ( $0-10$ years) and in late adulthood ( $>60$ years). More commonly, the diagnosis may be missed as a result of inadequate physical examination or laboratory evaluation, which can lead to unnecessary surgical procedures and delays to diagnosis and appropriate therapy.

The important aspect of the case presentation concerns the diagnostic delay: Qualitative psycho-oncological studies have documented that certain patients responded to disease symptoms by using self-medication or waiting. Problems with access to healthcare professionals and patients' social 
responsibilities served as the predominant barriers to prompt seeking assistance (7). As with the present case, the patient failed to pay attention to the groin enlargement until it grew so quickly that it became a burden. Therefore, it is assumed that the patient may neglect this important sign if the groin mass were not large enough. The present case demonstrated the importance of a thorough physical examination and consideration of testicular mass in the evaluation of patients presenting with neck nodes. Furthermore, this case was unusual in another aspect. The hugeness and massive metastasis of the primary lesion resulted in the consideration of possible lymphoma. The diagnosis of seminoma was established by immunohistochemistry.

Notably, once neck nodes are involved, the tumor is classed as stage 3 and initial treatment is generally chemotherapy. The majority of patients with this tumor stage are curable since the introduction of cisplatin-based chemotherapy, a regimen that has recently been suggested as a less toxic and equally effective alternative in intermediate prognosis metastatic seminoma (overall survival of $91 \%$ after 3.5 years) (8). In the present patient, chemotherapy yielded complete clinical remission. The present study was confused as to why the patient refused surgical resection of left orchid testis, which may be like a 'untimed bomb'. The present case highlighted that cryptorchidism remains the best established risk factor for testicular germ cell tumor and medical practitioners must remember to include metastatic testicular germ cell tumors in their differential diagnosis of supraclavicular neck mass.

\section{Acknowledgements}

The present study was funded by the Jilin Financial Foundation (no. 20157301051).

\section{References}

1. Aparicio J: SEOM clinical guidelines for diagnosis and treatment of testicular seminoma. Clin Transl Oncol 13: 560-564, 2011.

2. Winter $\mathrm{C}$ and Albers P: Testicular germ cell tumors: pathogenesis, diagnosis and treatment. Nat Rev Endocrinol 7: 43-53, 2011.

3. Reiter WJ,Brodowicz T, Alavi S,Zielinski CC, Kozak W, Maier U, Nöst G, Lipsky H, Marberger M and Kratzik C: Twelve-year experience with two courses of adjuvant single-agent carboplatin therapy for clinical stage I seminoma. J Clin Oncol 19: 101-104, 2001.

4. Pettersson A, Richiardi L, Nordenskjold A, Kaijser M and Akre O: Age at surgery for undescended testis and risk of testicular cancer. N Engl J Med 356: 1835-1841, 2007.

5. Carriere P, Baade P and Fritschi L: Population based incidence and age distribution of spermatocytic seminoma. J Urol 178: 125-128, 2007.

6. van Vledder MG, van der Hage JA, Kirkels WJ, Oosterhuis JW, Verhoef $\mathrm{C}$ and de Wilt JH: Cervical lymph node dissection for metastatic testicular cancer. Ann Surg Oncol 17: 1682-1687, 2010.

7. Malavasi N, Ferrara L, Fiorani C, Saviola A and Longo G: Diagnostic delay in oncology: A case report of metastatic seminoma. Case Rep Oncol 4: 216-221, 2011.

8. ASCO annul meeting proceedings (Post-Meeting Edition). J Clin Oncol 27: 5031, 2009. 